\title{
Design and Implementation of Magnetic Induction based Wireless Underground Communication System Supporting Distance Measurement
}

\author{
Min-Joon Kim ${ }^{1}$, Sung-Hun Chae ${ }^{1}$, Young-Bo Shim ${ }^{1}$, Dong-Hyun Lee ${ }^{1}$, Myung-Jin Kim ${ }^{1}$, \\ Yeon-Kug Moon ${ }^{1}$ and Kon-Woo Kwon ${ }^{2 *}$ \\ ${ }^{1}$ SoC Platform Research Center, Korea Electronics Technology Institute \\ Seongnam, Korea \\ [e-mail:\{mjoon, shchae, ybshim, donghyun2, kmjketi, ykmoon\}@keti.re.kr] \\ ${ }^{2}$ Department of Computer Engineering, Hongik University \\ Seoul, Korea \\ [e-mail: konwoo@hongik.ac.kr] \\ *Corresponding author: Kon-Woo Kwon
}

Received March 3, 2019; revised May 16, 2019; accepted June 6, 2019;

published August 31, 2019

\begin{abstract}
In this paper, we present our proposed magnetic induction based wireless communication system. The proposed system is designed to perform communication as well as distance measurement in underground environments. In order to improve the communication quality, we propose and implement the adaptive channel compensation technique. Based on the fact that the channel may be fast time-varying, we keep track of the channel status each time the data is received and accordingly compensate the channel coefficient for any change in the channel status. By using the proposed compensation technique, the developed platform can reliably communicate over distances of $10 \mathrm{~m}$ while the packet error rate is being maintained under 5\%. We also implement the distance measurement block that is useful for various applications that should promptly estimate the location of nearby nodes in communication. The distance between two nodes in communication is estimated by generating a table describing pairs of the magnetic signal strength and the corresponding distance. The experiment result shows that the platform can estimate the distance of a node located within $10 \mathrm{~m}$ range with the measurement error less than $50 \mathrm{~cm}$.
\end{abstract}

Keywords: underground communication, wireless underground sensor networks (WUSN), magnetic induction (MI), distance measurement.

A preliminary version of this paper was presented at ICONI 2018, and was selected as an outstanding paper. This work was supported by Institute for Information \& communications Technology Promotion(IITP) grant funded by the Korea government(MSIT) (No. 2018-0-01203, Development of Precise Positioning Sensor Module for Detection of Position-Change in Underground Facility) 


\section{Introduction}

Wireless underground sensor networks (WUSNs) have drawn significant attention as they can be used in many important industrial tasks such as fault detection of gas pipelines (see Fig. 1), smart agriculture for farms, and air pollution control in underground mines [1-3]. In order to perform these tasks successfully, it is important for a node of WUSN to be able to estimate the location of nearby nodes and reliably communicate with each of them. It is, however, challenging to achieve the accurate localization and robust wireless communication quality in underground environments. It is especially true when electromagnetic (EM) waves are used for underground communication because of following reasons. First, underground environments contain various sources for material absorption such as soil, rock, and water. Hence, in comparison to an over-the-air channel, the underground channel leads to the significantly higher levels of attenuation by reducing power density of EM waves. Second, it is difficult to predict the communication quality in underground environments. For example, an electrolyte dissolved in underground soil water is one type of sources that cause the path loss of EM waves. It should be noted that soil water content varies by location. Moreover, even for the samae location, water content may dynamically change from time to time. Thus, the amount of signal loss would depend on location and time. Accordingly, it is challenging to design the optimal wireless communication system based on EM waves in underground environments. Third, the inhomogeneous nature of underground environments causes the multi-path fading and thus negatively impacts communication with EM waves. For instance, roots of vascular plants may be randomly located underground and these make a change in the direction of EM waves, producing multi-path fading.
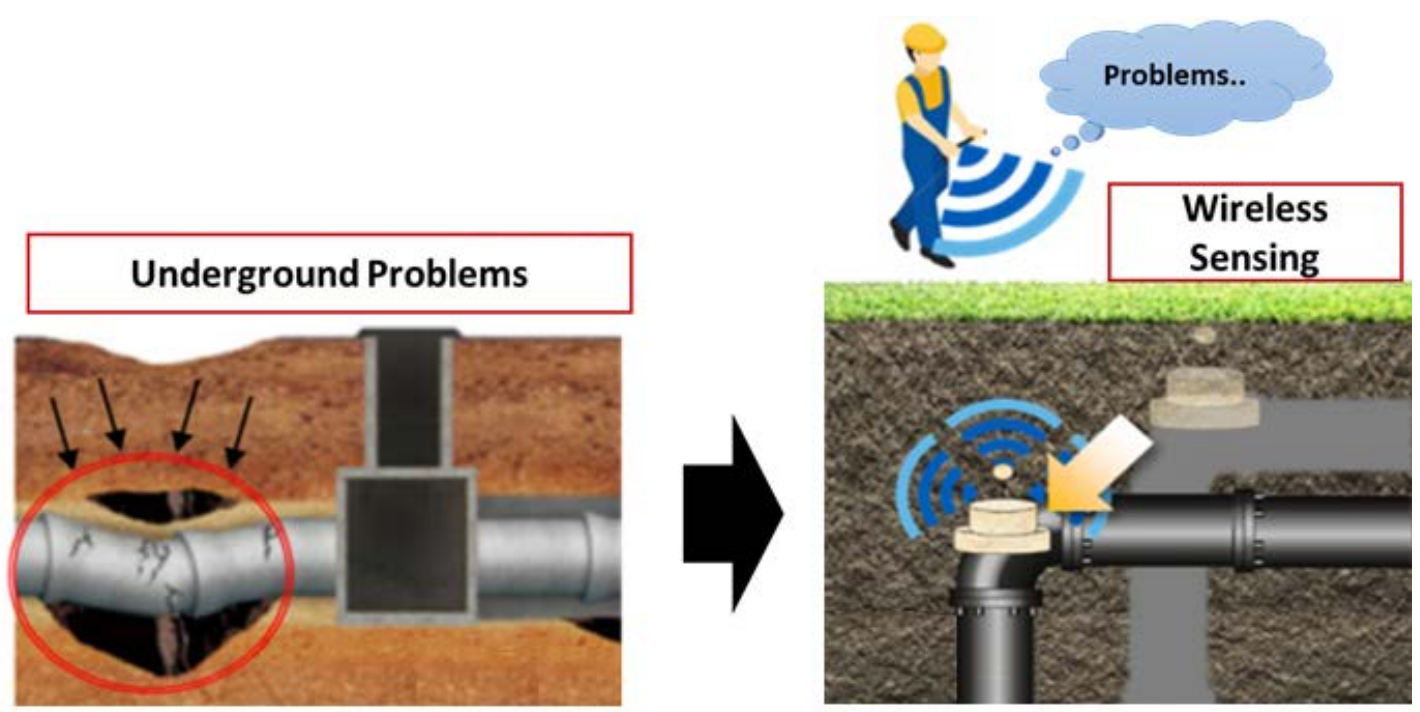

Fig. 1. Fault detection of gas pipelines using wireless underground sensor network.

In order to address the aforementioned issues of EM based underground communication, there have been active studies to find the alternative communication technique suited for WUSNs [4-6]. Amongst various techniques, the magnetic induction (MI) based technique is considered to be the leading candidate as it offers several benefits. First of all, the magnetic 
permeability of soil, rock, and water are almost identical to that of air. For this reason, MI based communication in underground environments causes little change in the rate of magnetic field attenuation from that through the air. Secondly, unlike most wireless communication approaches using far field, the MI technique generates the magnetic field in the near field. In other words, the MI based communication is non-propagating and thus does not produce multi-path fading. Based on such inherent advantages, many ideas and designs for WUSNs using MI technique have been proposed and analyzed [7-15]. Out of these approaches, however, most have been either theoretical analysis or simulation under ideal assumptions. In order to transfer such research ideas and analysis results into reality, there is a need to develop a testbed prototype to conduct validation in real environments. In this work, we develop a prototype that performs wireless communication as well as distance measurement in underground environments. We implement the wireless communication block using MI technique so as to fully utilize the aforementioned advantages of MI based underground communication. To improve the communication quality, we propose and implement the adaptive channel compensation technique. Based on the fact that the channel may be fast time-varying, we keep track of the channel status each time the data is received and adaptively compensate the channel coefficient for any change in the channel status. By using the proposed compensation technique, our developed platform can reliably communicate over distances of 10 meters while the packet error rate (PER) is being maintained under $5 \%$. We also implement the distance measurement block that is useful for various applications that should estimate the location of nearby nodes in communication. The distance between two nodes in communication is measured by generating a table describing pairs of the magnetic signal strength and the corresponding distance. The experiment result shows that the platform can estimate the distance of a node located within 10m range with the measurement error less than $50 \mathrm{~cm}$.

The contributions of this paper are three folds:

- First, we implement and validate the MI based WUSN platform that can perform wireless communication as well as distance measurement in real underground environments. Our developed WUSN platform includes the loop antenna, analog subsystem, and digital subsystem to form a complete system for performance evaluation. The antenna is designed with the carefully chosen parameters to transmit and receive signals at $128 \mathrm{KHz}$. The analog and digital subsystems are implemented together on the single package that includes Altera Cyclone-V Field Programmable Gate array (FPGA). We show that our platform can be used to validate the theoretical models of MI based WUSN.

- Second, we propose and implement the adaptive channel compensation in order to achieve the reliable communication in underground environment. Based on the fact that the channel may vary from time to time, we track the channel status each time the data is received and adaptively compensate the channel coefficient. Experiment results show that the proposed compensation technique can enable the communication range up to $10 \mathrm{~m}$ while the PER is maintained low under $5 \%$.

- Third, we add the distance measurement functionality in the proposed system so that the node acting as data receiver can easily estimate the location of the data transmitter. Basic idea is to convert the received signal strength to the distance between two nodes in communication. Based on theoretical analysis and field tests, 
we generate a lookup table describing pairs of the received signal strength and the corresponding distance. The table is stored and utilized to function the distance measurement promptly. Experiment results show that the implemented function can estimate the distance of a node located within $10 \mathrm{~m}$ range with the measurement error less than $50 \mathrm{~cm}$.

The rest of this paper is organized as follows. In Section 2, we discuss the related work in WUSN. Section 3 briefly reviews the wireless underground communication based on MI technique. Section 4 presents our proposed platform and Section 5 discusses experiment results. We conclude our work in Section 6.

\section{Related Work}

Researchers have studied how to successfully build WUSNs, where underground sensors can be utilized to perform a variety of industrial tasks such as gas leakage detection on pipelines, intelligent agriculture for farms, and air quality management in mines [1-3]. Ian F. Akyildiz et al. [4] showed the advantages of WUSNs over wired solutions. The authors also provided the design challenges for wireless communication in underground environments. Design challenges include power conservation, topology design, and antenna design. In [5] and [6], authors set up the theoretical channel models to discuss high path loss occurred when EM waves are used for underground communication. A. R. Silva et al. [7] demonstrated that EM techniques for underground communication actually experience the significantly high path loss by developing the testbed for validation in real environment.

To address the challenges in EM based underground communication, MI techniques were introduced in literatures. [8]-[15] showed that the MI based communication can be the reliable solution for underground environments since magnetic permeability of soil is similar to that of air. Since MI technique supports only short range of communication, there have been proposals to increase the communication range. For example, C. J. Stevens et al. [10] developed the concept of MI waveguide, whereas H. Guo et al. [11] developed the 3D MI communication model. Although these studies have shown that MI based techniques are promising solutions for building WUSNs in many applications, there is a strong need to develop a testbed in order to conduct validation in real underground environments. In this work, we develop the MI based WUSN platform that can be used for validation underground. The developed platform achieves the reliable communication quality via the adaptive channel compensation. Also, the platform includes the practical solution to measure the distance between nodes in communication by quantifying magnetic signal strength.

\section{Magnetic Induction based Communication}

This section briefly reviews the communication based on MI. Fig. 2 illustrates a transmitting (Tx) antenna and a receiving ( $\mathrm{Rx})$ antenna separated by the communication medium. As mentioned earlier, the impact by the communication medium is relatively small because the magnetic permeability of most natural materials underground is fairly identical to that of air. To support this fact, Table 1 shows that relative magnetic permeabilities of paramagnetic and diamagnetic materials are in the range between 0.999 and 1.001 . 


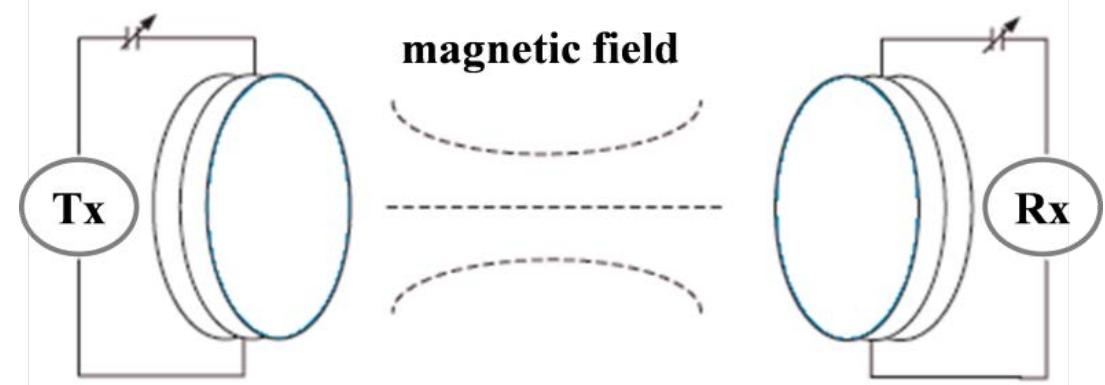

Fig. 2. Wireless communication based on magnetic induction.

Table 1. Relative permeability as the various material.

\begin{tabular}{|c|c|c|c|c|c|c|c|}
\hline \multirow{2}{*}{ Material } & \multirow{2}{*}{ Air } & \multicolumn{3}{|c|}{ Para-Magnetic } & \multicolumn{3}{c|}{ Dia-Magnetic } \\
\cline { 3 - 8 } & & Aluminum & Magnesium & Palladium & Copper & Silver & Gold \\
\hline \hline $\begin{array}{c}\text { Relative } \\
\text { Permeability }\end{array}$ & 1.0 & 1.000021 & 1.000012 & 1.000082 & 0.99999 & 0.9998 & 0.9996 \\
\hline
\end{tabular}

Tx and Rx antennas are essentially a coil loop of wire. It is worth noting that the communication channel gain increases as the coil size grows or the number of turns increases. The channel gain is also impacted by resistance per unit length of the coil loop. The lower the resistance per unit length, the higher the channel gain we can achieve. Hence, in order to achieve the high channel gain while maintaining the small antenna size, it is important to choose the wire with low resistivity (i.e., high conductivity).

MI wave propagation experiences the path loss. However, the path loss does not increase too rapidly as the communication distance increases. The following equation shows that the path loss $\left(L_{\mathrm{MI}}\right)$ is the logarithmic growth as follows:

$$
L_{\mathrm{MI}}=6.02+60 \log (r)+10 \log \left(\frac{N_{t}}{N_{r} a_{t}^{3} a_{r}^{3}}\right)
$$

where $r$ denotes the transmission distance, $N_{t}$ and $N_{r}$ are the number of truns of the coil for transmitter and receiver, resectively. Parameters $a_{t}$ and $a_{r}$ are the radii of the coil for transmitter and receiver, resectively.

In the case of the EM based communication [7], the path loss $\left(L_{\mathrm{EM}}\right)$ increases in a linear fashion in underground environments as follows.

$$
L_{\mathrm{EM}}=64+20 \log (r)+20 \log (\beta)+8.67 r \alpha,
$$

where $\alpha$ denotes the attenuation cost, $\beta$ denotes the phase shifting constant. For comparison, we perform the simulation of path loss versus distance for MI wave propagation and EM wave propagation with specific parameters: $\alpha=2, \beta=1, N_{t}=N_{r}$, and $a_{t}=a_{t}=0.2$. As shown in Fig. 3, the path loss of the MI wave propagation in soil medium is the logarithmically increasing function while the path loss of the EM wave propagation is the linearly increasing function. In terms of the robustness over path loss, the MI based communication is more suited to WUSN. 


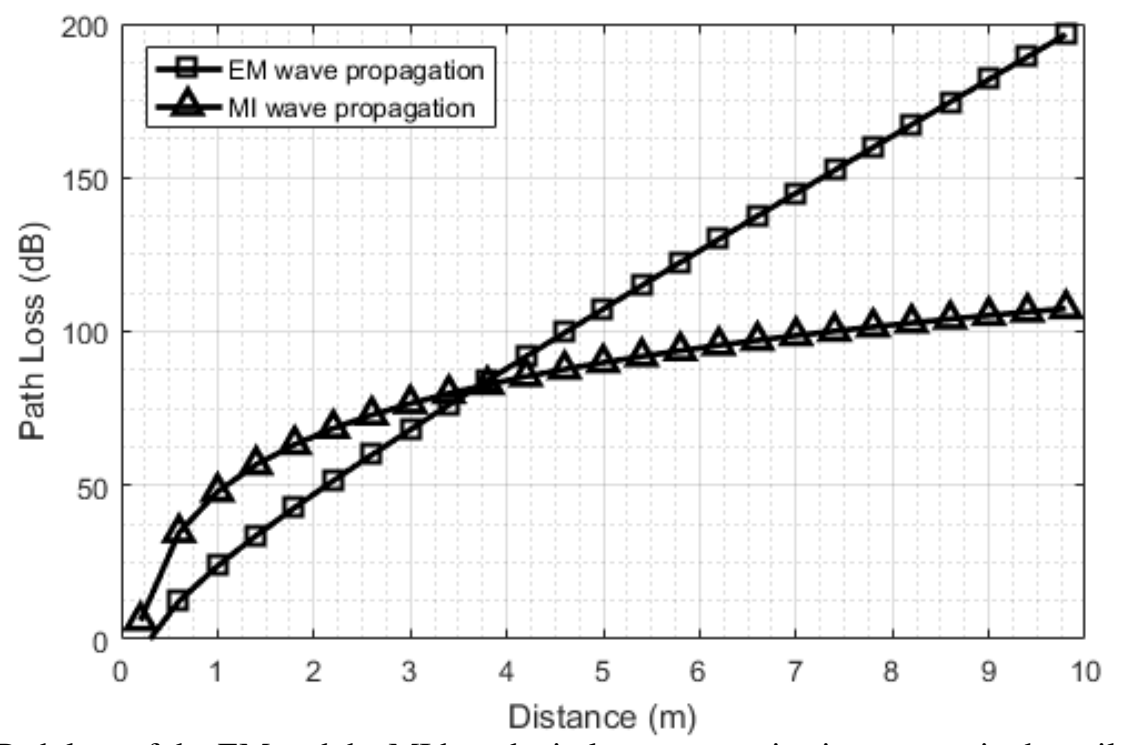

Fig. 3. Path loss of the EM and the MI based wireless communication system in the soil condition.

\section{Proposed Platform}

This section presents our developed platform for WUSN applications. The platform is designed to support MI-based communication as well as the functionality of distance measurement between two nodes in communication. The communication block includes the channel compensation technique to improve the communication quality. In the following subsection, we first introduce the overall system architecture then discuss individual blocks in details.

\subsection{System Architecture}

The system architecture is composed of multiple blocks including (1) loop antenna, (2) analog communication block, (3) digital communication block, (4) distance measurement block, and (5) sensor block, as shown in Fig. 4. The primary role of sensor block is to gather application-specific data. When there is a need to transmit the gathered sensor data via MI communication, the sensor data is first combined with control information such as preamble and header to form the complete network packet. Such packetization is followed by modulation that is performed by Tx block within digital communication block. The modulated data stream is further translated into the analog waveform by Tx block of analog communication block. Finally, the loop antenna is set to be Tx mode for sensor data transmission via near-field MI communication.

The process of receiving sensor data is performed in the reverse direction: the loop antenna in Rx mode receives the MI-based signals and passes them to analog communication's Rx block so that the received signals can be properly amplified, low-pass filtered and converted into the digitized signals. It is followed by demodulation and depacketization by Rx block in digital communication block. Note from Fig. 4 that the ADC output, which is digitized signal, is fed into the distance measurement block. The strength of the signal is translated into the path loss that is used to estimate the distance between two antennas. 


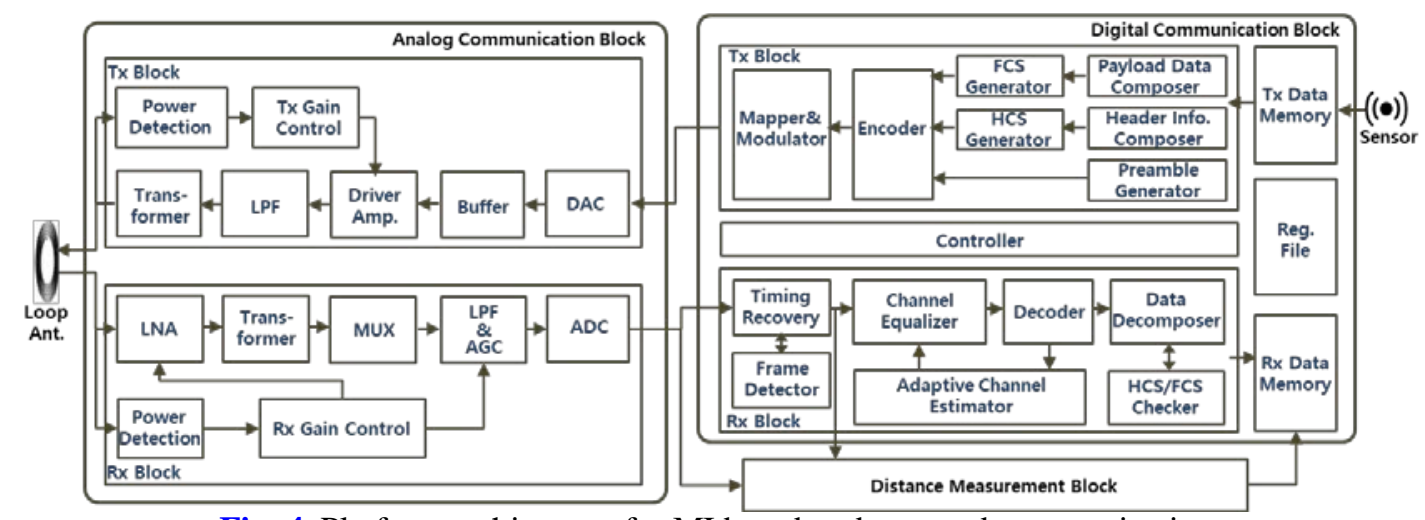

Fig. 4. Platform architecture for MI based underground communication.

The antenna is essentially a small coil loop of wire to transmit and receive signals based on magnetic field. As shown in Table 2, we consider two types of loop antennas, one in the shape of a circle and the other one of rectangle shape. In this work, antenna parameters including wire thickness, antenna size, and the coil turn numbers are carefully chosen to obtain its target resonant frequency of $128 \mathrm{KHz}$, as shown in Table 2.

Table 2. Configurations of the different shape of loop antennas.

\begin{tabular}{|c|c|c|}
\hline Parameter & Rectangular Antenna & Circle Antenna \\
\hline \hline \multirow{2}{*}{ Shape } & \multicolumn{2}{|c|}{} \\
\cline { 2 - 3 } & & \\
\hline Inductance & $96.7 \mathrm{uH}$ & $95.7 \mathrm{uH}$ \\
\hline Wire & litz wire & litz wire \\
\hline Size & $350 \mathrm{~mm} \times 230 \mathrm{~mm}$ & $\mathrm{R}=150 \mathrm{~mm}$ \\
\hline Turn Number & 11 & 17 \\
\hline Cap. & $4.7 \mathrm{nF} \times 3,2.2 \mathrm{nF} \times 1$ & 352 \\
\hline Q-Factor & 252 & $\mathrm{nF} \times 3,2.2 \mathrm{nF} \times 1$ \\
\hline
\end{tabular}

The analog and digital communication blocks are integrated into a single printed circuit board (PCB) as shown in Fig. 5. The analog communication block on the top left of the PCB includes multiple sub-blocks including low noise amplifier (LNA), transformer, multiplexer (MUX), low pass filer (LPF), analog-to-digital converter (ADC), and digital-to-analog converter (DAC). LNA is used in the first stage of Rx block to amplify the weak signals received from antenna while minimizing the noise components. The transformer in Rx block is used to generate two different waveforms in which one is in-phase (I) component and the other is quadrature (Q) component with 90-degree shift. Then, MUX is used to select one component, either I or Q, at a time. The selected component is passed to LPF with the cutoff frequency of $25 \mathrm{KHz}$. Before demodulating and depacketizing in the digital domain, the analog component needs to be converted into the discrete-time signal. In this work, we use 12-bit ADC within Rx block. For Tx operation, the analog communication block performs the 
inverse operation of the aforementioned Rx operation using DAC, buffer, driver amplifier, LPF, and transformer sub-block. The digital communication block is implemented in Altera Cyclone-V FPGA located on the top right of the PCB. Main functionalities of Tx in the digital communication block include the packetization, the digital encoding based on Manchester code, and the modulation using binary phase shift keying (BPSK) scheme. Main functionalities of Rx block include timing recovery, frame detection, Manchester decoding, and depacketization.

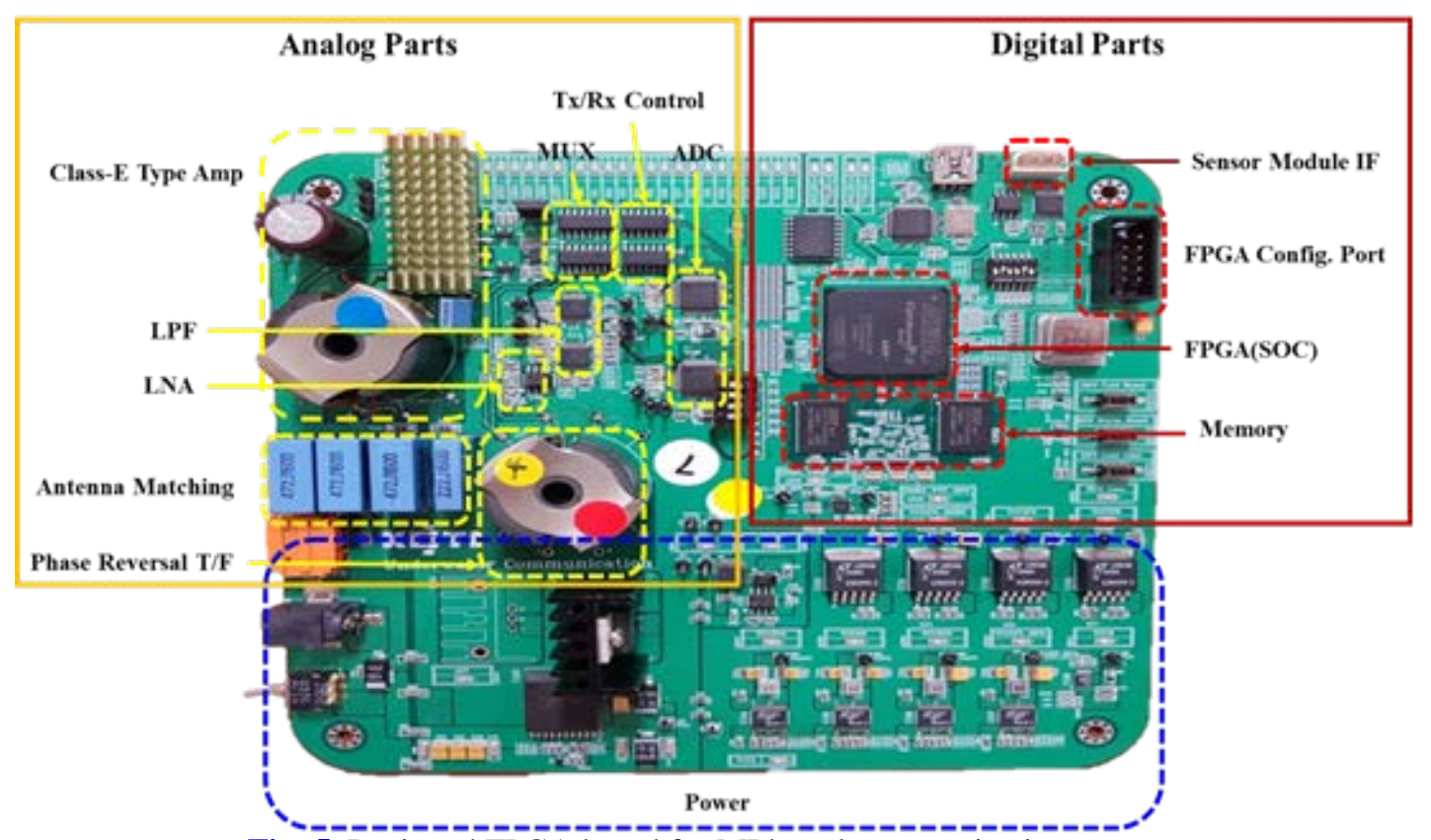

Fig. 5. Designed FPGA board for MI based communication system.

\subsection{Channel Estimation and Compensation}

In order to improve the communication performance in underground environments, we propose and implement the adaptive channel compensation technique. Our technique is based on the fact that WUSNs may experience the fast time-varying channel where the channel coefficient does not remain static until an entire single packet is delivered.

Channel compensation begins with observing the received signal vector $(y)$ that corresponds to the preamble if it is the first vector of the packet. Note that $y$ is the signal that passes through the channel. Hence, if the channel causes any change, $y$ is not necessarily identical to the preamble data vector $(p)$ known at the receiver. We estimate the channel by comparing $y$ and $p$. That is, the channel coefficient is calculated as the inner product of $y$ and $p$. For the next coming signal vector, we note that the channel coefficient is not guaranteed to remain static under fast time-varying channel. Hence, for each time the signal vector is received, the new coefficient is calculated as a weighted average of the earlier channel coefficient and the new comparison result based on the following equation:

$$
h_{n}=\left\{\begin{array}{cc}
y_{n-1}^{T} \mathbf{p} & \text { for preamble } \\
(1-\alpha) h_{n-1}+\alpha y_{n}^{T} x_{n} & \text { for data }
\end{array} .\right.
$$


where $h_{\mathrm{n}}$ and $y_{\mathrm{n}}$ denote the $n$-th estimated channel coefficient and received signal vector, respectively. $x_{\mathrm{n}}$ and $p$ denote transmitted signal vector and preamble data vector, respectively. $\alpha$ is the control parameter for the adaptation speed. Channel compensation procedure is summarized in the simplified block diagram of Fig. 6.

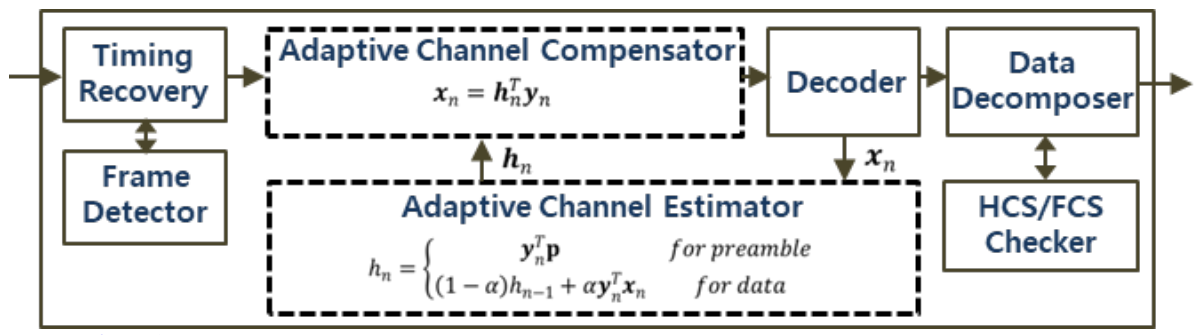

Fig. 6. Block diagram for the adaptive channel estimation and compensation.

\subsection{Distance Measurement}

We implement the distance measurement functionality in the proposed system so that the node acting as data receiver can estimate the location of the data transmitter. Basic idea is to convert the received signal strength indicator (RSSI) to the distance between two nodes in communication. It is noteworthy that the path loss is actually the more accurate indicator than RSSI to estimate the distance. It is because the distance is indicated by the transmitted signal strength as well as RSSI. Recall that the path loss is determined by considering both transmitted and received signal strengths. Hence, we first generate a table describing pairs of the RSSI and the corresponding path loss. Next, we generate the second table for pairs of the path loss and the corresponding distance. Both tables contain appropriate values based on theoretical analysis and field tests. Using two tables stored in FPGA memory block, the distance measurement works with three steps as shown in Fig. 7:

1) Path loss estimation: RSSI of each received signal is translated into the corresponding path loss by referring to the first table. We generate total 512 path losses for a single frame that is defined as a group containing 512 modulated signals.

2) Frame synthesis: a single representative path loss per frame is calculated by averageing 512 path losses.

3) Distance estimation: the representative path loss is translated into the corresponding distance by referring to the second table.

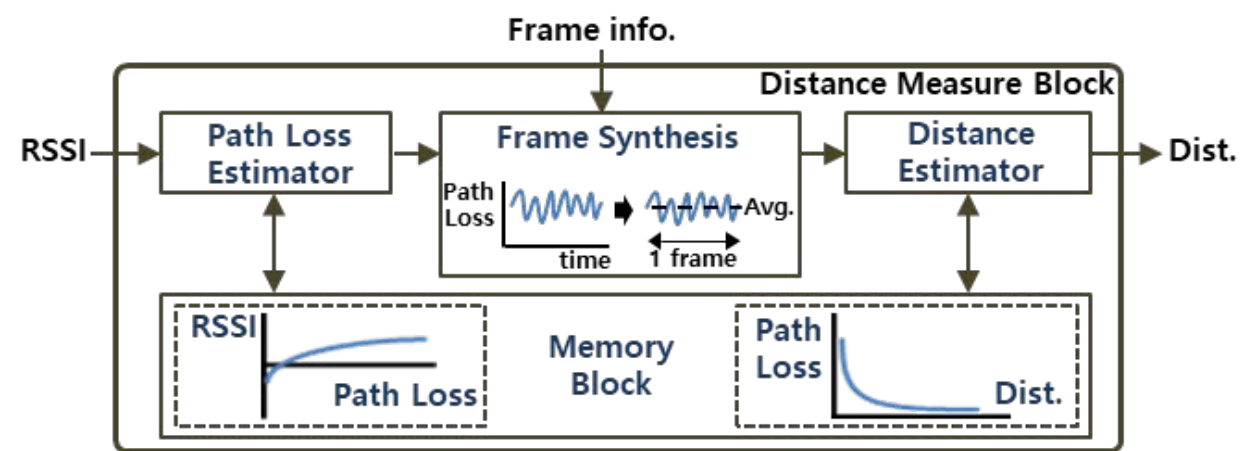

Fig. 7. Block diagram for the distance measurement. 


\section{Experiment Results}

This section presents our experiment result with the developed platform for WUSN. Experiment includes measurement of PER during communication and positioning accuracy during distance measurement. To perform the experiment in environments similar to real underground channel, we use the jumbo box where the developed platform is contained and the moist soil is filled for the remaining space. In the following subsection, we first introduce the experiment environment and process then discuss the experiment result.

\subsection{Experiment Environment}

To setup the experiment environment, we use two platforms with each being inside the separate jumbo box filled with moist soil. The platform including the loop antenna and the PCB module is packaged into a case that satisfies the international protection standard (IP 65) for dustproof and waterproof conditions. The jumbo box is made of plastic to minimize its impact on the magnetic field communication. Two platforms are located at a distance of 10 meters or longer.

We conducted two experiments that include PER and positioning accuracy to verify platform performance. For the PER test, we transmit more than 10,000 bits of dummy data at 4096 bps every 1m distance from the transmitter with two cases that applied our proposed adaptive channel compensator algorithm or not.

For the positioning performance test, the receiver is moved from $1 \mathrm{~m}$ to $20 \mathrm{~m}$ while the distance information according to the ADC signal level is received through the LED of the positioning test board connected to the receiver.

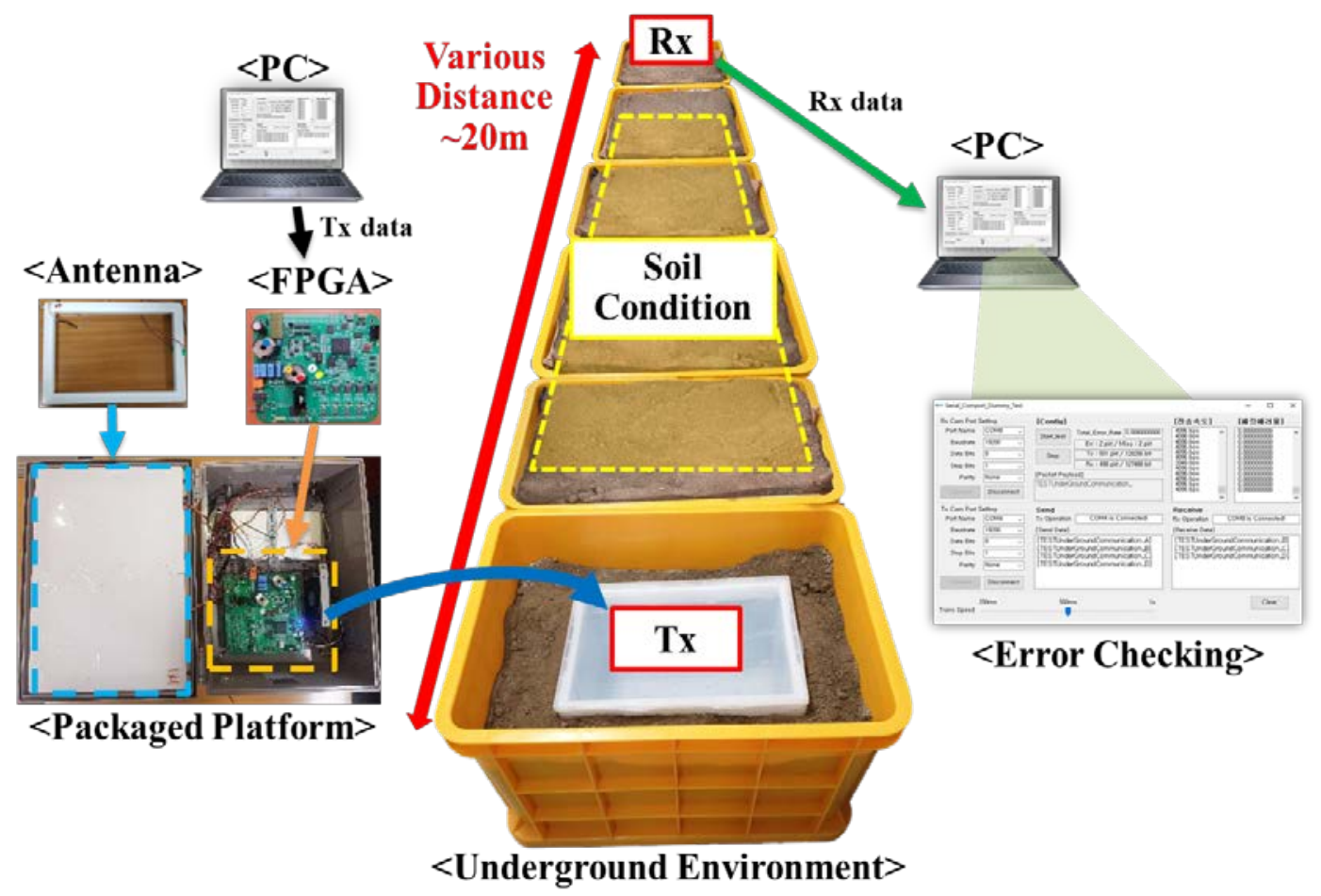

Fig. 8. The experiment environment for MI based underground communication 


\subsection{Evaluation Results}

The communication performance was evaluated by checking PER at different distances. The evaluation result is shown in Fig. 9. The proposed algorithm, the adaptive channel estimator and compensator in Sec. 4.2, can achieve 5\% of PER at $10 \mathrm{~m}$ distance and $10 \%$ of PER at $12 \mathrm{~m}$, while the conventional channel equalizer only can achieve $5 \mathrm{~m}$ distance at PER $5 \%$ and $7 \mathrm{~m}$ at PER 10\%. This result shows that the proposed adaptive channel estimation and compensation algorithm makes the platform more robust to path loss.

\begin{tabular}{|c|c|c|}
\hline $\begin{array}{c}\text { Receive } \\
\text { Algorithm }\end{array}$ & $\begin{array}{c}\text { Achievable } \\
\text { Distance } \\
\text { (PER 5\%) }\end{array}$ & $\begin{array}{c}\text { Achievable } \\
\text { Distance } \\
\text { (PER 10\%) }\end{array}$ \\
\hline $\begin{array}{c}\text { Without adaptive } \\
\text { channel } \\
\text { compensator }\end{array}$ & $5 \mathrm{~m}$ & $7 \mathrm{~m}$ \\
\hline $\begin{array}{c}\text { With adaptive } \\
\text { channel } \\
\text { compensator }\end{array}$ & $10 \mathrm{~m}$ & $12 \mathrm{~m}$ \\
\hline
\end{tabular}

(a)

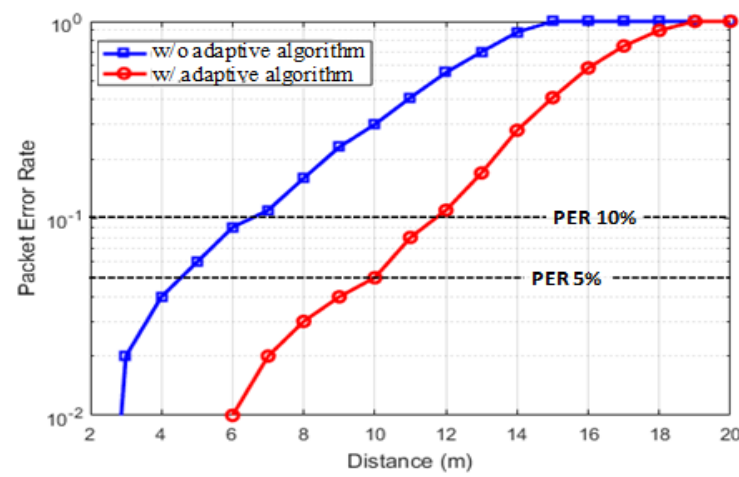

(b)

Fig. 9. Evaluation results of the error rate performance with and without adaptive channel compensator;

(a) Achievable distance, and (b) PER versus distance

The distance measurement performance was evaluated by checking the estimated and the real distance between Tx and Rx. As shown in Fig. 10 (a), the LED shows up the estimated distance and we summarized the results from 20 times of experiment at the same distance in Fig. 10 (b). At $10 \mathrm{~m}$ of distance between $\mathrm{Tx}$ and $\mathrm{Rx}$, the distance was estimated by the proposed platform within $50 \mathrm{~cm}$ error. At $20 \mathrm{~m}$ of distance, the error range increases up to $3 \mathrm{~m}$.

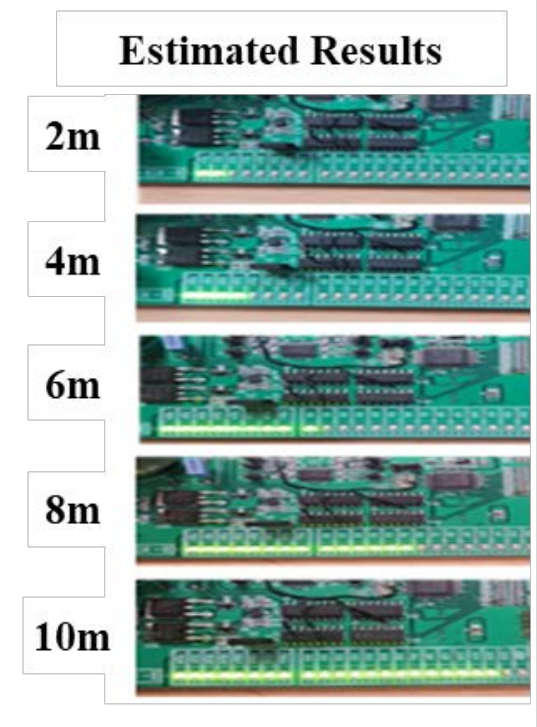

(a)

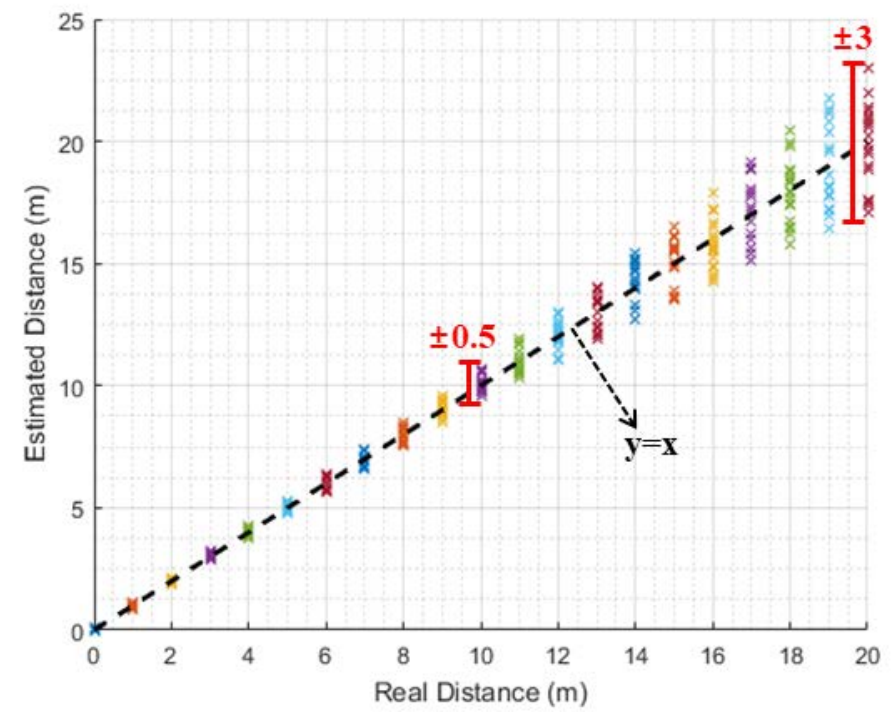

(b)

Fig. 10. Evaluation results of the distance measurement; (a) LED show-up as the estimated distance, and (b) the estimated versus the real distance 


\section{Conclusion}

MI based communication have been studied as an emerging and promising research area to enable an efficient wireless communication for the WUSNs. Since the propagation medium such as soil, concrete brings high path loss to the traditional EM communication in the underground environments, the EM communication does not work well and can be only applied for very small transmission ranges. Although MI based communications work at low-frequency so that it only supports low data rates, it is expected to be enough to establish the various applications for WUSNs.

In this paper, we designed the wireless communication platform based on MI for WUSN systems. The platform is designed to perform MI communication as well as distance measurement using MI signal. The main contributions of this paper are that 1) we implement and validate the MI based WUSN platform, 2) we propose and implement the adaptive channel estimation and compensation technique, and 3) we add the distance measurement functionality to be enable to use widely in the WUSN systems.

In order to verify the platform, the experiment was conducted with our packaged platform under the soil condition. At first, the PER was measured to check the communication performance. The result shows our platform can achieve $10 \mathrm{~m}$ distance under $5 \%$ of PER with the proposed adaptive channel estimation and compensation technique while it only achieved $5 \mathrm{~m}$ distance with the conventional technique. Secondly, the estimated distance was measured as the increasing distance. The result shows our platform can estimate $10 \mathrm{~m}$ distance within $50 \mathrm{~cm}$ error.

\section{References}

[1] Xin Tan, Zhi Sun, and Ian F. Akyildiz, "Wireless Underground Sensor Networks: MI-based communication systems for underground applications," IEEE Antennas and Propagation Magazine, vol. 57, no. 4, pp. 74-87, August, 2015. Article (CrossRef Link)

[2] Vehbi C. Gungor and Gerhard P. Hancke, "Industrial Wireless Sensor Networks: Challenges, Design Principles, and Technical Approaches," IEEE Transactions on Industrial Electronics, vol. 56, no. 10, pp.4258-4265, October, 2009. Article (CrossRef Link)

[3] Emad Felemban, Faisal Karim Shaikh, UmairMujtaba Qureshi, Adil A. Sheikh and Saad Bin Qaisar, "Underwater Sensor Network Applications: A Comprehensive Survey," International Journal of Distributed Sensor Networks, vol. 11, no. 11, pp. 1-14, November, 2015. Article (CrossRef Link)

[4] Ian F. Akyildiz, Pu Wang and Zhi Sun H., "Realizing underwater communication through magnetic induction," IEEE Communications Magazine, vol. 53, no. 11, pp. 42-48, November, 2015. Article (CrossRef Link)

[5] Niaz Ahmed, Andriy Radchenko, David Pommerenke and Yahong Rosa Zheng, "Design and Evaluation of Low-Cost and Energy-Efficient Magneto-Inductive Sensor Nodes for Wireless Sensor Networks," IEEE Systems Journal, vol. 13, no. 2, pp. 1135-1144, June, 2019. Article (CrossRef Link)

[6] Agnelo R. Silva and Mahta Moghaddam, "Design and Implementation of Low-Power and Mid-Range Magnetic-Induction-Based Wireless Underground Sensor Networks," IEEE Transactions on Instrumentation and Measurement, vol. 65, no. 4, pp.821-835, November, 2015. Article (CrossRef Link)

[7] Steven Kisseleff, Ian F. Akyildiz and Wolfgang H. Gerstacker, "Digital Signal Transmission in Magnetic Induction Based Wireless Underground Sensor Networks," IEEE Transactions on Industrial Electronics, vol. 63, no. 6, pp. 2300-2311, June, 2015. Article (CrossRef Link) 
[8] Ashish Kumar Sharma, Sadanand Yadav, Sandeep N. Dandu, Vinay Kumar, Joydeep Sengupta, Sanjay B. Dhok and Sudhir Kumar, "Magnetic Induction-Based Non-Conventional Media Communications: A Review," IEEE SENSORS JOURNAL, vol. 17, no. 4, pp. 926-940, February, 2017. Article (CrossRef Link)

[9] Steven Kisseleff, Ian F. Akyildiz and Wolfgang Gerstacker, "On Modulation for Magnetic Induction based Transmission in Wireless Underground Sensor Networks," in Proc. of IEEE International Conference on Communications(ICC), pp. 71-76, May, 2014.

Article (CrossRef Link)

[10] Zhi Sun and Ian F. Akyildiz, "Magnetic Induction Communications for Wireless Underground Sensor Networks," IEEE Transactions on Antennas and Propagation, vol. 58, no. 7, pp. 2426-2435, July, 2010. Article (CrossRef Link)

[11] Hongzhi Guo and Zhi Sun, "Demo Abstract: Prototyping M $^{2}$ I Communication System for Underground and Underwater Networks," in Proc. of IEEE Conference on Computer Communications Workshops (INFOCOM WKSHPS), pp. 962-963, May, 2017.

Article (CrossRef Link)

[12] Shih-Chun Lin, Ian F. Akyildiz, Pu Wang and Zhi Sun, "Distributed Cross-Layer Protocol Design for Magnetic Induction Communication in Wireless Underground Sensor Networks," IEEE Transactions on Wireless Communications, vol. 14, no. 7, pp. 4006-4019, March, 2015. Article (CrossRef Link)

[13] Hong Wu, Xuefeng Jiang, Pengfei $\mathrm{Xu}$ and Chao Zhang, "Efficient Integration of Magnetic Positioning and Stable Communications," IEEE Magnetics Letters, vol. 9, pp.1-4, July, 2018. Article (CrossRef Link)

[14] Olutosin Charles Fawole and Massood Tabib-Azar, "An Electromechanically Modulated Permanent Magnet Antenna for Wireless Communication in Harsh Electromagnetic Environments," IEEE Transactions on Antennas and Propagation, vol. 65, no. 12, pp.6927-6936, October, 2017. Article (CrossRef Link)

[15] Vinay Kumar, Rutuja Bhusari, Sanjay B. Dhok, Arun Prakash, Rajeev Tripathi and Sudarshan Tiwari, "Design of Magnetic Induction Based Energy-Efficient WSNs for Nonconventional Media Using Multilayer Transmitter-Enabled Novel Energy Model," IEEE Systems Journal, vol. 13, no. 2, pp. 1285-1296, July, 2018. Article (CrossRef Link)
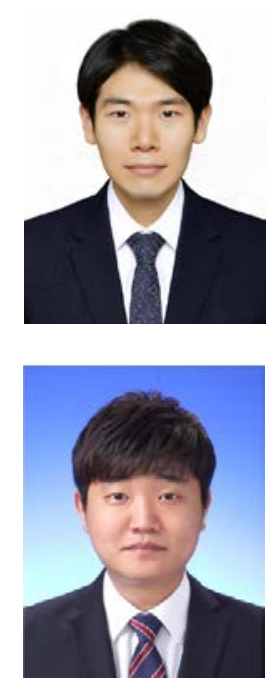

Minjoon Kim received the B.S. and Ph.D. degree in electrical and electronic engineering from Yonsei University, Seoul, Korea, in 2012 and 2018, respectively. He is currently a senior researcher in the Korea Electronics Technology Institute, Korea. His research interests include digital signal processing algorithm and SoC/VLSI implementation for wireless communication systems.

Sung Hun Chae received his B.Sc. and M.Sc. in Electrical Engineering from Kookmin University, Korea in 2012 and 2014. Currently he is working towards researcher in the Korea Electronics Technology Institute, Korea. His research interests include MI based wireless communication, High precision positioning system, QoS provisioning, Mobility management and Femtocell networks. 


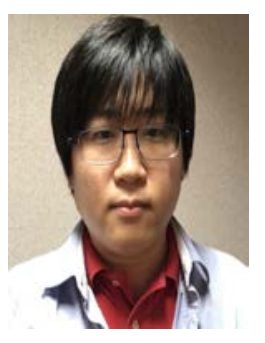

Young Bo Shim obtained his Ph.D. in physics with longitudinal-mode controlled lasers and application to imaging system from Hanyang University (Korea) in 2017. He is currently engaged as a researcher in the Korea Electronics Technology Institute, Korea. His major interests are Lidar systems, imaging, and ranging techniques.

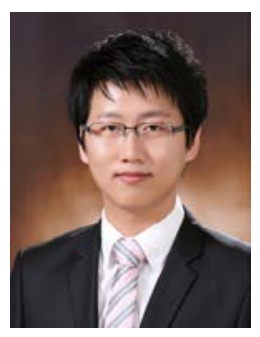

Dong-Hyun Lee received a B.S. and M.S. degree in Electronic Engineering from Dong-Guk University, Korea, in 2010 and 2012, respectively. Since 2012, he has worked for Korea Electronic Technology Institute. His major interests are high precision positioning system, RF ranging algorithm, wireless communication network.

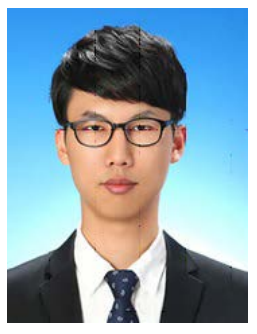

Myoung-Jin Kim received his B.S. and M.S degrees in Sejong University, Seoul, Korea, in 2015 and 2017 respectively. Since 2017 he has been a resercher of Korea Electronics Technology Institute (KETI), Gyeonggi-do, Korea. His research interests include wireless communication systems design and High precision positioning system.

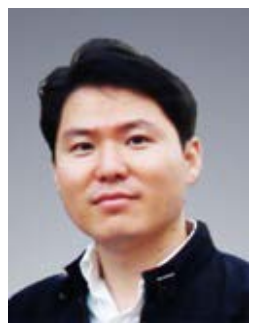

Yeon-Kug Moon received a B.S. and M.S. degree in Electronic Engineering from In-ha University, Korea, in 1998 and 2000, and Ph.D. degree in BioMicrosystem Technology from Korea University, Korea, in 2014, respectively. From 2002 to 2005, he worked for Samsung Electronics Company. Since 2005, he has worked for Korea Electronic Technology Institute. His major interests are Lidar system, localization, and SoC Platform.

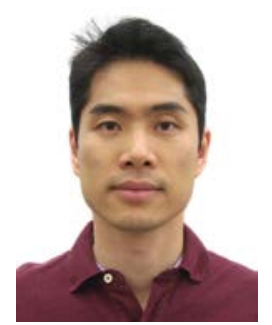

Kon-woo Kwon received the B.S. and M.S. degrees in electrical engineering from Korea University, Seoul, South Korea, in 2007 and 2009, respectively, and Ph.D. degree in electrical and computer engineering from Purdue University, West Lafayette, IN, USA, in 2015. He is an Assistant Professor at the Department of Computer Engineering, Hongik University, Seoul, South Korea. He was a Component Design Engineer at Intel Corporation from 2015 to 2018. He was also with Hyundai Motor Company, Seoul, South Korea, as a Research Engineer from 2009 to 2010. His research interests include low power circuit and scalable architecture design. 